\title{
Lafcadio Hearn oder die Welt als Archipel
}

Kommen wir zum letzten Beispiel einer global aufgestellten Reiseliteratur im 19. Jahrhundert, wobei sich in dieser Figur das 20. Jahrhundert schon sehr deutlich ankündigt. Der am 27. Juli 1850 auf der griechischen Insel Lefkas (Santa Maura) geborene Patricio Lafcadio Tessima Carlos Hearn, der am 26. September 1904 unter seinem später angenommenen japanischen Namen Koizumi Yakumo in Tokio verstarb, darf aus einer transarealen Perspektivik sicherlich als einer der interessantesten und schillerndsten Autoren an der Wende zum 20. Jahrhundert gelten. Die Biographie von Lafcadio Hearn ist für sein Leben zweifellos symbolhaft: Sie führte ihn vom griechischen Archipel über Irland und England, Cincinnati, New York und New Orleans in die Karibik und schließlich nach Nippon, gleichsam ein Weg von Insel zu Insel, von Archipel zu Archipel, der sich auch in seinen Reiseberichten niederschlug.

Doch wir wollen uns Lafcadio Hearn nicht als jenem Autor annähern, der wohl wie kaum ein anderer das Bild prägte, das man sich nach der Jahrhundertwende von Japan und dem japanischen Archipel im Abendland machte. Vielmehr soll er uns als ein reiseliterarischer Schriftsteller erscheinen, der wohl wie kaum ein anderer die archipelische und transarchipelische - andere Archipele weltweit einschließende - Welt der Karibik entdeckt hat und literarisch zum Leben erweckte. Lafcadio Hearn ist auf diesem Gebiet sicherlich eine der faszinierendsten Figuren, bei der Reisen und Schreiben intim aufeinander abgestimmt sind.

In seinen Texten wird die Vielverbundenheit dieser Welt voller Inseln und Inselwelten auf anschauliche Weise vor Augen geführt, zugleich aber auch zu hören gegeben, erscheinen doch in seiner komplexen, fast rhizomatisch sich ständig verzweigenden Sprache all jene Idiome der Karibik, aber auch der mit ihnen verbundenen Archipele weltweit, die uns Hearn zu Ohren Gehör bringt. All diese Sprachen, all diese Klänge, all diese Stimmungsnuancen führen zu jener vielstimmigen Melodie und Tonalität, deren karibischer Rhythmus einen so antillanischen Autoren und Musikologen wie den Kubaner Alejo Carpentier sehr begeisterte.

Doch kommen wir zu Hearns Reisebericht aus der Karibik. In seinem auf zwei sehr unterschiedliche Reisen in die Welt der Antillen zurückgehenden und erstmals 1890 veröffentlichten Reisebericht Two Years in the French West Indies hat Lafcadio Hearn seine höchst dynamische, mobile Form des Schreibens aus der Bewegung zur schriftstellerischen Perfektion gebracht. Bei dieser archipelartig aus kürzeren und längeren Texten bestehenden Sammlung narrativer Texte handelt es sich nicht nur um ein Bravourstück poetischer Prosa, die den Figurationen und Konfigurationen der Inselwelten adäquat ist, sondern im

Ә Open Access. (C) 2020 Ottmar Ette, publiziert von De Gruyter. (cc) BY-NC-ND Dieses Werk ist lizenziert unter der Creative Commons Attribution-NonCommericial-NoDerivatives 4.0 Lizenz.

https://doi.org/10.1515/9783110650686-026 


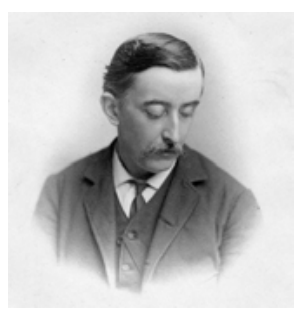

Abb. 87: Lafcadio Hearn alias Koizumi Yakumo (Lefkada, 1850 Tokyo, 1904).

stärksten Sinne um ein Schreiben, das die Welt der Inseln sowohl präsentiert als auch literarisch repräsentiert.

Lafcadio Hearn legt dabei einen reiseliterarischen Text sui generis vor, der uns von den Erlebnissen des Ich-Erzählers auf den französischen Antillen berichtet; in jenem 'Westindien', in welchem sich die karibischen und amerikanischen mit den afrikanischen, europäischen und asiatischen Kulturen, Sprachen und Lebensformen auf intime Weise verknüpfen, ohne doch miteinander zu verschmelzen. Dabei war Hearn gleichsam sein eigener Reisemaler, hatte er doch eine Menge Geld in Ausrüstung und Training mit seinen photographischen Apparaten investiert, ${ }^{1}$ mit deren Hilfe er wirklich unvergessliche Zeugnisse aus der Lebenswelt der Karibik zum Zeitpunkt seiner Reise machte. Hearn war gewiss nicht der erste Reiseschriftsteller, der die noch etwas neuartige Photographie, die gleichwohl ihren Kinderschuhen entwachsen war, zu seinen Gunsten einsetzte. Aber er tat es mit viel künstlerischem Fingerspitzengefühl.

Lafcadio Hearn gelang es, seine nicht nur polyseme, sondern weit mehr noch polylogische Schreibweise immer wieder fensterartig auf transarchipelische Dimensionen hin zu öffnen, um dadurch die ganze Welt wie in einem Insel-Fraktal einfangen und abbilden zu können. Der Trumpf einer derartigen Schreibweise war es, die für jede Insel wiederum spezifischen weltweiten Beziehungen zur Darstellung zu bringen und damit das Portrait einer Welt zu liefern, die nicht kontinuierlich, sondern diskontinuierlich, aber zugleich hochgradig vernetzt miteinander verbunden ist. Denn wer Insel sagt - dies bestätigte Hearns Schreiben sehr gut -, der meint eine Kartographie, die weltweiten Zuschnitts ist.

Lafcadio Hearns Reisetext bezieht seine literarische Einheit gerade aus seiner mobilen Vielgestaltigkeit, ist Bewegung doch auf allen Ebenen das Hauptmovens

1 Zur Geschichte dieser teuren Photoausrüstung vgl. die „Note on the Texts“ in Hearn, Lafcadio: American Writings. Hg. von Christopher Benfey. Library of America, No. 190. Des Moines New York: Library of America 2009, S. 828. 
seines Schreibens. Die Erzählprozesse in Two Years in the French West Indies entfalten sich auf unterschiedlichen Ebenen und in verschiedenartigen Textsorten, zeigen aber häufig eine mise en abîme-Struktur, in welcher eine bestimmte Erzählung für den gesamten Text steht und zugleich andere Erzählkerne an sich zu binden vermag. Man könnte bei seinen Texten von einer offenen, mangrovenartigen Strukturierung sprechen, die sich nicht um eine zentrale, sondern um viele dezentrale Wurzeln schart und aus diesen eine ungeheure Kraft saugt.

Die unterschiedlichsten geologischen wie biopolitischen Dimensionen erscheinen in diesen Reisetexten und geben uns einen Eindruck von der Vielgestaltigkeit einer Welt, die aus Inseln gemacht ist. So unternimmt Lafcadio Hearn in diesem polyperspektivischen Reisebericht des Öfteren erfolgreich den Versuch, die geographischen und geologischen mit kulturellen Aspekten auf eine Weise $\mathrm{zu}$ verbinden, die uns verstehen lässt, dass wir auf unserem Weg durch die Karibik zugleich auf unterschiedlichen Routen durch eine zutiefst transarchipelische Welt unterwegs sind. Unser Weg ist dabei hochgradig diskontinuierlich und könnte zu jedwedem Zeitpunkt abbrechen oder an anderer Stelle wieder aufgenommen werden. Eine itinerarische Struktur betrifft nur die grobe Anordnung, wird auf der Ebene der reiseliterarischen Durchführung aber aufgelöst.

So etwa ausgehend von einer Beschreibung und Darstellung der Montagne Pelée, des Vulkanriesen auf der kleinen französischen Insel Martinique, in einer Prosa, die uns den Gegenstand selbst schon nahebringt. Überall tauchen Inselstrukturen auf, überall sind Natur und Kultur eng miteinander verflochten:

But its centre is not one enormous pyramidal mass like that of 'La Montagne': it is marked only by a group of five remarkable porphyritic cones; - the Pitons of Carbeit; - while Pelée, dominating everything, and filling the north, presents an aspect and occupies an area scarcely inferior to those of Aetna.

-Sometimes, while looking at La Pelée, I have wondered if the enterprise of the great Japanese painter who made the Hundred Views of Fusiyama could not be imitated by some creole artist equally proud of his native hills, and fearless of the heart of the plains or the snakes of the slopes. A hundred views of Pelee might certainly be made: For the enormous mass is omnipresent to dwellers in the northern part of the island and can be seen from the heights of the most souther mornes. It is visible from almost any part of St. Pierre, - wich nestles in a fold of rocky skirts. It overlooks all the island ranges, and overtops the mighty Pitons of Carbet by a thouseand feet [... . ${ }^{2}$

Eine Insel verbirgt eine andere, ein Vulkan macht uns auf einen anderen Vulkan aufmerksam. In einer für Lafcadio Hearns Schreiben typischen Bewegung

2 Hearn, Lafcadio: „Two Years in the French West Indies.“ In (ders.): American Writings, S. 387 . 
fokussiert die Erzählerfigur zunächst ihren Gegenstand, die Montagne Pelée, um deren Zentrum sogleich wieder zu relativieren und zu relationieren. Denn so, wie die Insel in der Karibik mit einer Insel im Mittelmeer verbunden wird, so verweist die Montagné Pelée auf den Ätna, der auf Sizilien aufragt und der Insel ihre Struktur liefert. Lafcadios Heimatregion, die griechische Inselwelt, ist da nicht weit.

Doch damit nicht genug: Denn noch fehlt die weltumspannende Konfiguration des Archipelischen. Die sich anschließende Bewegung öffnet die transatlantische und zugleich transarchipelische Relation sogleich mit Blick auf die japanische Inselwelt, welche ebenfalls die Übereinstimmung eines Zusammenspiels zwischen Meer, Eiland und Vulkan bietet. Dabei wird die perfekte Silhouette des Fujiyama jedoch nicht unter geologischen oder vulkanologischen, sondern unter künstlerischen und ästhetischen Aspekten in den Text eingeführt.

Dafür muss Hearn ein wenig den Unwissenden spielen. Der diskrete Verweis auf den im Text namentlich nicht genannten großen japanischen Maler Katsushika Hokusai (1760-1849) und dessen berühmte Farbholzschnitt-Serie der Darstellungen des Fujiyama macht es Hearn möglich, einen fiktiven kreolischen Maler ins Spiel zu bringen, der ähnlich wie der japanische Künstler ein polyperspektivisches Werk schaffen könnte. Es wäre ein künstlerisches Werk, das aus den Bewegungen rund um die zerfurchte und von Abertausenden von Schlangen 'verseuchte' Vulkanregion der Montagne Pelée hervorgehen müsste. Diese Vulkanregion kannte Lafcadio Hearn ausgezeichnet, hatte er sie doch mehrfach durchwandert und immer wieder auch photographiert. Er durchstreifte sie unermüdlich ausgehend von der damaligen Hauptstadt St. Pierre, das einem Ausbruch der Montagne Pelée später zum Opfer fiel.

Die Photographie ist Hearns treuer Begleiter auf der Reise. Aber der intermediale Verweis von Hearns Schrift auf Hokusais Kunst schließt auf transmedialer Ebene eine immanente Poetik des eigenen Reisens, Schreibens und Photographierens mit ein. Denn das von ihm geforderte polyperspektivische, der Serie des großen japanischen Künstlers nachempfundene Werk hat - wie es der Text auch in dieser Passage vorführt - Lafcadio Hearn fraglos zumindest auf der literarischen Ebene selbst geschaffen. Wie bei Cézanne ergibt sich ein polyperspektivisches Verhältnis zwischen Künstler und (von ihm unendliche Male portraitierten) Berg.

Diese implizite immanente Poetik, die hier ausgehend von den transarchipelischen Beziehungen der Montagne Pelée zu Ätna und Fujiyama entfaltet wird, bezieht sich auf die literarischen Skizzen des Schriftstellers griechisch-irischer Herkunft selbst. Denn er ist es, der im Grunde längst zu jenem scheinbar namenlosen kreolischen Maler geworden ist, der uns immer wieder neue Ansichten vor Augen führt. Der Berg erscheint in diesen Ansichten - wie bei Cézanne - auf eine kubistische Weise, die gleichsam künstlerisch zum damaligen Zeitpunkt bereits in der Luft liegt. Aus der Polyperspektivität wird eine Polyrelationalität 
hervorgezaubert, die eine auf den ersten Blick bisweilen verwirrende, in labyrinthischen Sätzen sich entfaltende Bilderfolge entstehen lässt. Es ist eine Bilderfolge, welche einerseits die ständigen Veränderungen der Perspektive aufzeigt, andererseits aber sich ineinander projiziert und ein kubistisches Bildnis ergibt. Mit einem von Georg Forster und nach ihm Alexander von Humboldt geprägten Ausdruck könnte man von 'Ansichten der Natur' sprechen.

Diese vielperspektivischen Ansichten gelten auch für das Künstlersubjekt, für den Reisenden selbst. Aus dem Griechen, Iren, Briten und US-Amerikaner wird ein Antillaner und letztlich auch Japaner, obwohl diese Phase seines Lebens zum damaligen Zeitpunkt eigentlich noch vor ihm liegt. Seine vielen Namen füllen jenen Ort des namenlosen kreolischen Malers aus, weil in jenem scheinbar 'leer' bleibenden Ort des Namens viele Namen, viele Orte transarchipelisch eingetragen werden können, ja eingetragen werden müssen. Das Konstruktionsprinzip ist auch hier das Fraktal, das auf einer internen strukturellen Selbstähnlichkeit beruht und auf eine ganze Welt von Inseln und Vulkanen verweist. Auch eine sogleich noch anzuführende Passage wird belegen: Es ist, als ob in diesem großen Vulkan der Insel Martinique die Vulkane dieser Welt, ja die unterschiedlichsten Regionen dieser Erde zusammenliefen, ohne hier doch ihr 'Zentrum', ihren Mittelpunkt zu finden. Es gibt kein Zentrum, gibt keinen Mittelpunkt - ganz so, wie die Mangroven keine gemeinsame Hauptwurzel besitzen. Denn ein solches Zentrum, einen derartigen Mittelpunkt der Erde kann für den Schriftsteller und Photographen Lafcadio Hearn gar nicht existieren.

Hearns Reisebericht Two Years in the French West Indies beginnt mit den Impressionen einer Schiffsreise an Bord jenes „langen, schmalen, grazilen Dampfschiffs aus Stahl“ ${ }^{3}$ mit dessen gleichsam photographischem Bild an Pier 49 im Hafen von New York der literarische Reisebericht einsetzt. Er beginnt damit mit dem Abschied vom Eigenen, um sich schrittweise dem 'Anderen' anzunähern und jene Veränderungen Punkt für Punkt aufzuzeigen, die sich auf dem Reiseweg ergeben. Wir haben es mit der literarischen Inszenierung einer Annäherung zu tun, in der die Tropenwelt voller Leben erscheint: Alles ist von „einen Sinn von WeltLeben (world-life) “4 erfüllt.

Hierbei ist die von Hearn gewählte Perspektive des reisenden Ich entscheidend. Dafür wird die Sichtweise eines nicht den Tropen entstammenden weißen Mannes von Beginn an eingenommen und markiert, eine Tatsache, die den Ich-Erzähler im weiteren Fortgang selbstverständlich nicht daran hindert, uns über lange Seiten hinweg detailreiche und eindringliche Alltagsbilder vom

3 Ebda., S. 159.

4 Ebda., S. $163 \mathrm{f}$. 
Leben der Wäscherinnen (blanchisseuses) oder der Trägerinnen schwerer Lasten (porteuses) aus Martinique zu liefern. Auch die entsprechenden Bilder steuert dieses reisende und photographierende Ich gleich mit bei. LebensBilder alltagskultureller Praktiken entstehen, wie wir sie in anderen Texten jenes Zeitraums in dieser Intensität wohl kaum noch einmal finden dürften. Lafcadio Hearn liefert uns ebenso reiseliterarische wie lichterzeugte Bilder, die bei ihm ein transmediales Netzwerk bilden. Reisen und Schreiben werden durch das Photographieren zu einem Dreieck ergänzt.

Im männlichen Blick werden die anfangs noch unbekannten Frauen zu wesentlichen Protagonistinnen des Insel-Lebens. Denn es sind gerade die Frauen, die uns ihr Lebenswissen und gleichsam ihre Geheimnisse mitteilen: Wir erfahren, wie lange sie arbeiten, wann sie essen, wie sie sich ihre Mahlzeiten zubereiten, aber auch, was für ein Leben sie sich erträumen - bis hin zu jener Gestalt einer jungen Frau, die auf der Rückreise an Bord kommt, um in New York als Hausangestellte $\mathrm{zu}$ arbeiten. Alle Schwierigkeiten, alle Hoffnungen, alle Illusionen werden vor unseren Augen ausgebreitet: Ein Wissen vom Leben auf der Insel wird uns ausgehend von den Frauen nahegebracht.

So sind es die porteuses, welche uns erzählen, welch schwere Lasten sie schon als junge Trägerinnen zu bewältigen gelernt haben, auf welchen Wegen sie die unterschiedlichen Teile der Insel miteinander verbinden, aber auch, welche Lieder sie singen, welchen Gefahren sie ausgesetzt sind, welchen Hoffnungen sie sich hingeben und welchen Träumen sie nachhängen. So entstehen Lebens-Bilder von höchster Eindringlichkeit. Und zugleich ein Lebenswissen und Überlebenswissen, das in seinem Kern auch ein Zusammenlebenswissen enthält: Denn Konvivenz ist ein hoher Wert, auf dessen Suche sich der Ich-Erzähler gemacht hat.

Die Reise selbst als Annäherung an die Tropen wird als rite de passage anschaulich gemacht. Der Übergang in die Tropen wird aus der Bewegung des Schiffes heraus als Passage in eine andere Farben- und Klangwelt, in eine andere materielle Sinnlichkeit wie in eine andere (und spirituelle) Traumlandschaft in den Klängen und Farben der Sprache ästhetisch und aisthetisch erfahrbar gemacht. Der Klang dieser sehr spezifischen Schreibweise, die häufig von Sätzen im français créole Martiniques durchbrochen ist, bedient sich des gesamten Registers der Klangfiguren, die in verschiedenen Sprachen eingeblendet werden. Nirgendwo ist die Sprache der verdoppelten Figur des Ich-Erzählers so dicht und plastisch wie in diesen Passagen einer langsamen Initiation.

Immer wieder treten neue Figuren, im literarischen wie im photographischen Bild, vor die Augen des Lesepublikums. Die Lebens-Bilder der mulattischen Wäscherinnen, die in St. Pierre ihre Wäsche ausbreiten, oder der Familien von Coolies, die ihre aus (Ost-) Indien mitgebrachten Normen und Formen des Zusammenlebens erläutern, prägen sich dank ihrer Lebendigkeit bei der Lektüre 
unmittelbar ein. Gerade die Rolle und Funktion der Coolies ist für den griechischen Briten aus den USA ein entscheidendes Element für die Darstellung der migratorischen Dynamik auf den Inseln.

Wir erfahren manches über die gesellschaftliche Realität der Inseln, vor allem aber über die gelebte Gemeinschaft von Eilanden, die ihre je eigenen Formen von Kleidung und Kochkunst, von Konvivenz und Konfliktivität entwickelt haben: Martinique ist zugleich eine Insel-Welt für sich und eine global vernetzte Inselwelt. Das karibische Eiland ist für Lafcadio Hearn die globale Insel par excellence. Diese Eigen-Logik einer Insel-Welt ist als hochverdichtete semantische Kippfigur stets auch eine Inselwelt im transarchipelischen, die Inselwelt der Antillen überschreitenden Sinne. Hearns lyrische Prosa führt uns dies immer wieder eindrucksvoll vor Augen und Ohren. Es zählt sicherlich zu den größten Verdiensten der verschlungenen, bisweilen labyrinthischen Schreibweise von Lafcadio Hearn, diese in der Tat hochkomplexe Bewegungsstruktur in ihrer ganzen Vektorizität, in all ihren Bahnungen und Bewegungen, ästhetisch überzeugend dargestellt zu haben. Hearns Schreiben ist, mit einem Wort, ein archipelisches Schreiben.

In einer weiteren, intertextuell spannenden Passage, die einen Bezug zu den längst klassisch gewordenen französischen Texten des Père Labat wie des Père Dutertre, aber auch zu der Abhandlung der Martinique gewidmeten Etudes historiques des - wie Hearn ihn nennt - kreolischen Autors Dr. E. Rufz ${ }^{5}$ herstellt, wird die Berg- und Vulkanwelt der französischen Antilleninsel erneut zum Ausgangspunkt einer die Tropen weltweit umspannenden Sichtweise. Nichts auf diesen Inseln ist lediglich auf eine einzige Insel bezogen.

Dies wird bei einer Bergbesteigung, gleichsam in der Aufwärtsbewegung einer seit Petrarca ${ }^{6}$ mit der Verstehens- und Erkenntnisbewegung verbundenen Gewinnung an Transparenz, ${ }^{7}$ deutlich skizziert. Folgen wir also der Dynamik des reisenden Ich:

With the diminuition of the wamth provoked by the exertion of climbing, you can begin to notice how cool it feels; - you could almost doubt the testimony ofyour latitude. Directly east is Senegambia: we are well south of Timbuctoo and the Sahara, - on a line with southern India. The ocean has cooled thewind; at this altitude the rarity of the air is northern; but in the valleys below the vegetation is African. The best alimentary plants, the best forage, the flowers of the gardens, are of Guinea; - the graceful date palms are

5 Vgl. hierzu ebda., S. 420.

6 Vgl. hierzu die klassische Studie von Ritter, Joachim: Landschaft. Zur Funktion des Ästhetischen in der modernen Gesellschaft. In (ders.): Subjektivität. Sechs Aufsätze. Frankfurt am Main: Suhrkamp 1989, S. 141-163.

7 Vgl. hierzu Starobinski, Jean: Jean-Jacques Rousseau. La transparence et l'obstacle. Suivi de Sept Essais sur Rousseau. Paris: Gallimard 1971. 
form the Atlas region: those tamarinds, whose thick shade stifles all other vegetable beneath it, are from Senegal. Only, in the touch of the air, the vapory colors of distance, the shapes of the hills, there is something not of Africa: that strange fascination which has given to the island its poetic creole name, - le Pays de Revenants. ${ }^{8}$

In dieser Passage wird bis ins Detail, in einer der Pflanzengeographie Alexander von Humboldts analogen Weise und wie in einem 'Naturgemälde der Tropenländer' die Herkunft jedes einzelnen Elements der Landschaft nachgezeichnet und als Ergebnis eines Migrationsprozesses charakterisiert. Die Rückkehr des Ich zu einem längeren Aufenthalt auf die Insel Martinique wird deutlich dadurch motiviert, dass sich von hier aus nicht allein eine Insel als eine gesamte, in sich abgeschlossene und zugleich vollständige Welt öffnet, sondern dass diese so eigene Welt zugleich wie in einem lebendigen Netzwerk die unterschiedlichsten Elemente eines weltweiten Beziehungsgeflechts in sich zu bündeln vermag. Martinique ist ein Mikrokosmos, eine InselInsel, ${ }^{9}$ die die Welt auf ihre eigene Weise enthält und gerade dadurch so eigen ist - eine transareal verdichtete Welt, auf der Ebene der Natur wie auf jener des Anbaus, der Kultur. Martinique ist, wir wiederholen uns, die globale Insel schlechthin.

So steht die französische Antilleninsel am Kreuzungspunkt und im Beziehungsgeflecht zwischen Westindien und Ostindien, zwischen den Indias occidentales und den Indias orientales, wie dies die Benennungen in der ersten Phase beschleunigter Globalisierung auszudrücken pflegten. Columbus hätte seine wahre Freude an dieser Zwischen-Insel zwischen der Alten Welt, aus der er kam, und der Alten Welt, in die er wollte, auf dem Boden jener Neuen Welt, deren Existenz er zu Lebzeiten niemals mehr bekräftigen und anerkennen wollte.

Die Insel um die Montagne Pelée ist eine globale und zugleich transareal vernetzte Insel. Sie steht in den 1890 in Buchform vorgelegten Two Years in the French West Indies - und hier spielt der reiseliterarische Text begrifflich schon im Titel die lange und komplexe Globalisierungsgeschichte aus - für die drei Phasen beschleunigter Globalisierung ein: zwischen den schwarzen Sklaven aus Afrika und den Coolies Asiens, zwischen den Bewohnern Europas und Ozeaniens bürgt sie für Vielverbundenheit und relationale Polylogik. Wir haben es mit einem Insel-Fraktal zu tun.

Kein Zufall also, dass der Reisende von New York, dem Zentrum der ersten außereuropäischen Globalisierungsmacht, ausgehend seine Reise in die Karibik

\footnotetext{
8 Hearn, Lafcadio: Two Years in the French West Indies, S. 419.

9 Vgl. hierzu Ette, Ottmar: Insulare ZwischenWelten der Literatur. Inseln, Archipele und Atolle aus transarealer Perspektive. In: Wilkens, Anna E. / Ramponi, Patrick / Wendt, Helge (Hg.): Inseln und Archipele: Kulturelle Figuren des Insularen zwischen Isolation und Entgrenzung. Bielefeld: transcript 2011, S. 13-56.
} 
auf einem Dampfschiff aus Stahl, auf einem „long, narrow, graceful steel steamer“10 unternimmt. Man könnte in diesem eleganten Dampfschiff einen gewiss nicht gänzlich unbeteiligten Boten jener New Steel Navy erblicken, welche die USA noch im selben Jahrzehnt zur unbestrittenen Führungsmacht auf dem Kontinent kanonieren und die spanische Flotte vor Manila und Santiago de Cuba im Meer versenken sollte. Dies ist die Vorschau auf eine Epoche, für welche diese Reiseliteratur von Lafcadio Hearn vielleicht wie keine andere steht: für die dritte Phase beschleunigter Globalisierung, deren militärisch-politische Höhepunkte sie gleichsam durch ihre Bewegungen und ihre Transportmittel ankündigt.

Auch auf einer demographisch-bevölkerungstechnischen, und damit im eigentlichen Sinne biopolitischen Ebene kann Martinique gleich für die verschiedenen Phasen beschleunigter Globalisierung einstehen. Das bis heute im Besitz Frankreichs, der Führungsmacht der zweiten Globalisierungsphase befindliche Martinique ist als Antilleninsel Teil jener Zone verdichtetster Globalisierung, die von der ersten Phase dieses Prozesses an die Menschen, Kulturen und Sprachen der europäischen 'Entdecker', Eroberer und Kolonisatoren, der unterschiedlichen Kulturen zugehörigen indigenen Bevölkerung sowie der hierher deportierten schwarzen Sklaven aus verschiedenen Teilen West- und Zentralafrikas, später aber auch der Kontraktarbeiter aus unterschiedlichen Regionen Asiens, beziehungsweise der Coolies aus Indien, aufeinander treffen und mehr noch aufeinander prallen ließ. In dem von Lafcadio Hearn entworfenen literarischen Landschaftsbild von Martinique fügen sich die so unterschiedlichen Elemente verschiedener Erdteile zu etwas Anderem, zu etwas Neuem, zu einem nicht mit Europa oder Afrika oder Asien zu verwechselnden Ganzen zusammen.

Dies wunderbar in seinem Reisebericht erfasst und durchbuchstabiert $\mathrm{zu}$ haben, ist sicherlich eines der größten Verdienste dieses reiseliterarischen Textes, der auf ideale Weise vom 19. ins 20. Jahrhundert überleitet. Lafcadio Hearns literarischer Reisebericht liefert uns für diese historischen, kulturellen und biopolitischen Vorgänge aus der Wechselbeziehung des Vorgefundenen, des Erfundenen und des Erlebten wie Gelebten das faszinierende Bewegungs- und Denkmodell. In seiner Vektorizität finden sich alle Vektoren der Vergangenheit, aber auch der Gegenwart und Zukunft wieder, welche uns den Raum der Antillen als einen transtropischen Bewegungs-Raum nahebringen. Die Vektoren der Reisen Lafcadio Hearns aber zeigten in den asiatischen Raum, hin $\mathrm{zu}$ jenem japanischen Archipel, auf welchem sich sein Leben schließlich runden und abschließen sollte.

10 Hearn, Lafcadio: Two Years in the French West Indies, S. 159. 